\title{
A pragmatist's approach to pathology costing: the Welsh Datatree project
}

\author{
D I Gozzard, M E Macaulay, D S Nuttall, E R M Jones
}

\section{Introduction}

Following the introduction of the National Health Service and Community Care Act (1990), it became apparent that the pathology services in the United Kingdom would have to function in a more commercial manner. This participation in the "open market" was, for the most part unexpected. Several "resource management sites" had been established at major hospitals in England with consequent injection of information technology and accompanying financial expertise. However, most pathology managers (usually medically qualified consultants) and the financial officers associated with their hospital units were unprepared for the amount of costing information required of them. In the past there had been little work carried out on costing in the NHS because there was no perception of the need for such data, although microcomputer software to assist costing had been described. ${ }^{1}$

One stimulus to obtain the necessary information was the tasking of the National Audit Commission to look at the "value-for-money" aspect of pathology services. ${ }^{2}$ To this end the Commission developed their own software and methodology of costing. These programs required certain data items from the laboratories, but often this information was diffcult to obtain, primarily because of lack of information technology and a paucity of firm financial information at the laboratory level. However, what has finally emerged is a "snapshot" of the pathology services from which comparisons can be made against the national average for a number of costed tests in the four main pathology disciplines of haematology and blood transfusion, clinical chemistry, microbiology and histopathology.

But this exercise was imposed on the pathology services with the pre-defined goal of obtaining information pertinent to "value", such as why should the blood count 10 miles away at hospital $X$ cost $50 \%$ more than at hospital Y? Although the final report highlighted potential problems, it produced little data of day-to-day managerial use. Since an axiom of systems analysis is to "define your outputs before your inputs" we might ask what information is required by pathologists with regard to pathology costing?

\section{What costing information is required}

Why cost at all? At present NHS hospitals allocate an annual budget to their laboratory service. Traditionally this has been a "top- down" budget into which we squeeze the demands on the service. Unfortunately the laboratories are demand-led, with pathologists having little control over the increasing requirements of the clinical services for pathology investigations. Our haematology laboratory has seen the annual number of test requests rise by $3 \cdot 4 \%$ in $1989-90$ and by $10 \%$ in 1990-91. Because these services have been supplied without charge to the purchasing clinical services, "market forces" have had no part to play in controlling laboratory use. The introduction of the "purchaser-provider" roles with contractual obligations has altered all this. General practitioners who have recently elected to manage their own budgets can now request laboratory tests wherever they want. Suddenly there is the need for information about laboratory test costs so that budgets for the use of the laboratory by these primary care doctors can be determined. The possibility of clinical budgeting in the hospital service is yet another situation when the laboratory will be expected to provide costing information, this time to clinicians.

Is test based costing information needed? A simple way to calculate the "average test cost" in a laboratory would be to divide the annual budget by the annual number of requests. In haematology a figure of $£ 2.52$ was obtained by this method. Is this reasonable? An obvious fallacy is that this costing process takes no account of the case-mix of the tests requested. The cost of a blood count may be near this figure but certainly the cost of isotopes for vitaminology tests or blood volume studies raises the costs of these tests to a different magnitude. Any increase in the requesting patterns of these costly tests would not be recouped by the "average test cost". So a form of weighting of the tests is required, such that an increase in the cost of a simple test requiring few extra resources is mirrored by a low test cost; an increase in a more complex test, or one that contains costly consumables, would be accounted for by a suitably higher test cost. Some form of banding of test costs would be a start to overcoming this difficulty, although this does not totally remove the risk of underresourcing with an increase in workload volume, particularly if the bands are few and relatively wide.

So do we need to know individual test costs? Although it has been suggested that this practice should be avoided, this is primarily on grounds of the necessity for frequent recalculation as prices of consumables or salaries 
change. ${ }^{3}$ Indeed, it was suggested that if individual test costs were required then they should mirror those prices published by private pathology laboratories. However, the Audit Commission has demonstrated the difference between NHS laboratory costs and private laboratory prices of the commoner high volume pathology tests. The difference in price may be for recapitalising of the laboratory, but are the NHS laboratories going to be allowed to adopt this process? It seems extremely doubtful at present because it would necessitate the carrying over of profits for several years to accrue the necessary budget for purchase of new equipment. A possible solution would be for the districts to set up capital funding programmes for the purchase of new laboratory equipment. This is already in place and working well in some districts.

Any information on costing will have a profound effect on the running of our laboratories. Once the costs of individual tests are known will this have any effect on test requesting? If a tariff of costs is available who will have the right of access to such information? We have seen locally that the releasing of a fixed price for a haematology test $(£ 2 \cdot 52)$ to our single budget holding primary care health centre has reduced the number of requests from that practice by $10 \%$. Other sites have shown a similar reduction. ${ }^{4}$ If a single average test price is released the fall in requests might be expected to be across the entire range of tests. But if a tariff of prices is released showing the cost to the practice of individual tests then a fall in the more expensive tests might be anticipated, increasing unit costs considerably and leading to a further increase in prices.

The NHS white paper Workinq for Patients (Cmnd 555) introduced the concept that managers should be encouraged to make the most efficient use of their physical resources by recognising that the continuing use of those resources has a cost. If the management of a laboratory is to be efficient then it should be able to play the "what if...?" with costings. One situation is the possible rationalisation of tests with one hospital performing a high cost, low volume test for several surrounding hospitals, thus reducing its unit cost. Different hospitals could specialise in different types of tests without the necessity for centralisation of all high cost tests. However, the requirements for this would be that, firstly, the costs of the tests are known at both the provider and the purchaser hospital and, secondly, that the capacity of the provider hospital for a centralised test is sufficient to take on the extra work.

Manpower is the main resource in all pathology disciplines contributing significantly to the cost of tests. With new technology the skill mix of the laboratory may change with obvious but obscure effects on costs. The introduction of new technology and new tests into the repertoire of the laboratory needs to be able to be costed in the light of available workload. Certainly it is these types of argument on financial as well as quality grounds that are going to unlock funds for purchase of new equipment.

\section{What costing information is available}

To cost laboratory tests rigorously requires up to date and accurate financial data-unfortunately not always available on a regular basis in the NHS. How many laboratories see their invoices, for example, to check if the discount arranged on the basis of early payment has occurred? Was payment delayed for some reason with a subsequent higher price for the commodity than anticipated? Close cooperation between the financial officers and the pathology managers is required for exchange of necessary information. Invoices must be checked by the laboratory staff so that the true costs of consumables can be captured.

Salary information can be obtained either from the end of year budget statement or direct from the finance officers. However, all oncosts must be included. Even so, there still exists the problem of building into the costing system a method to allow for pay rises and for probable backdating of the pay awards.

Capital depreciation in the Health Service has never been fully defined, and it has usually been the opportunity for a replacement machine that defines the life of any replaced equipment. Few districts have had the foresight to set up capital programs for replacement of pathology equipment. So the amortisation period has been rather haphazard and variable between pieces of equipment and between laboratories.

The introduction of capital charges has changed all this. The definition of a capital asset is "a tangible asset which is capable of being used in an authority's activities for a period which could exceed one year." Each laboratory will have an asset register containing the equipment deemed to be affected by capital charges. Although the calculations are complicated, there should be individualised capital charges available for each piece of equipment. This yearly charge can be input into the laboratory costing package.

\section{The pragmatic approach to costing}

For several years Welsh pathology laboratories have been interested in workload measurements. It was not suprising, therefore, that with the introduction of the NHS white paper and the changes that it suggested in the relationship between pathology laboratories and their purchasers, that a project should be established to investigate the costing of pathology. Rudimentary costing methods were presented at a joint meeting with representatives from the Welsh Office and the "value-formoney" unit, a directorate of the Welsh Office in early 1991. At this meeting pathologists from Glan Clwyd Hospital presented the Datatree costing program. This is a commercial costing package used in manufacturing and industry for several years, but at that time with no applications in the NHS. The meeting was impressed at the potential of the Datatree 
An example of built up costs using the Datatree costing method.

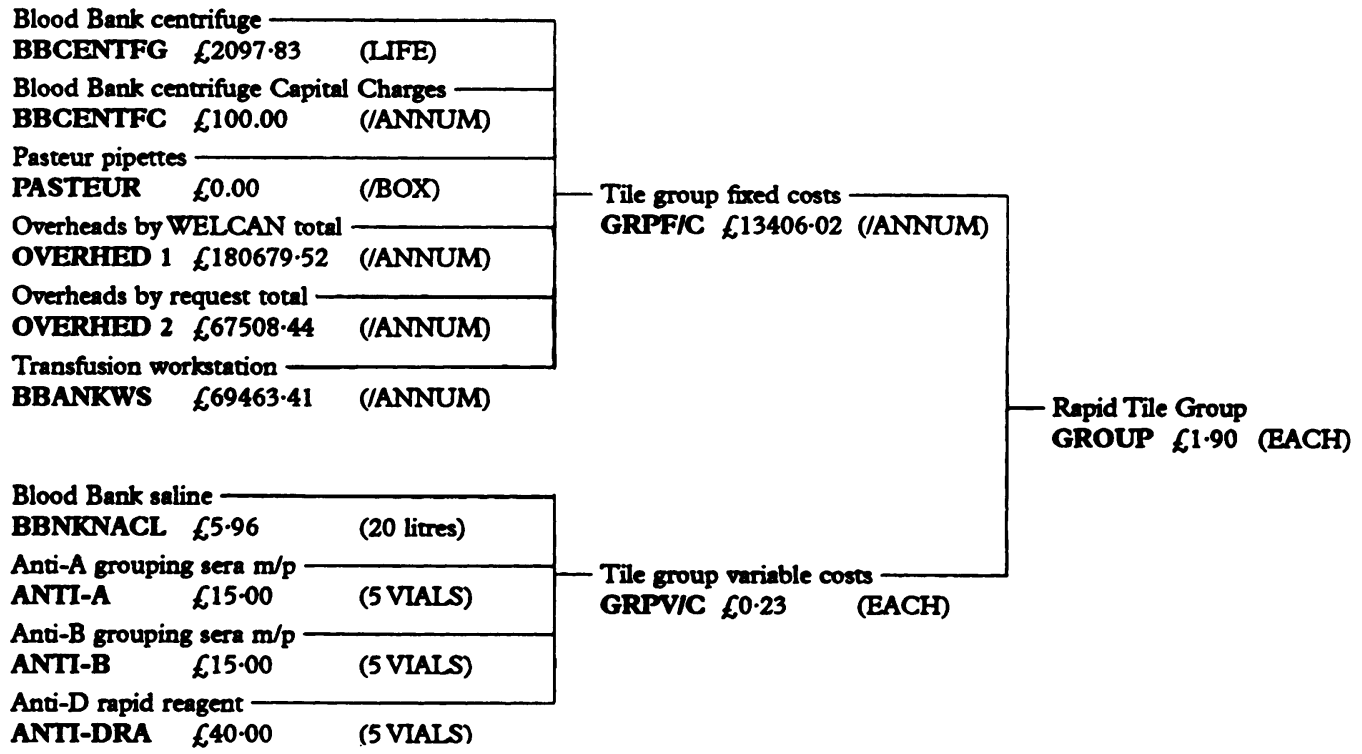

costing system and the Welsh Datatree Pathology Costing Project was established in mid1991.

The terms of reference were to "investigate whether the Datatree costing package can be utilised as a robust, volume-sensitive workcomplexity-weighted costing package across the pathology disciplines." The project team consisted of a representative from each of the four pathology disciplines and an accountant. The project was presented to Welsh pathologists in late 1991 and accepted by the Welsh Office as a possible standard for pathology costing in Wales. Although the software is not under discussion in this article, the development of the costing methods for the individual specialties unearthed problems that necessitated differences in approach across the disciplines.

The Datatree pathology costing method For each specialty the general approach to costing was the same, with division of costs into two main groupings, fixed and variable. Fixed costs, as defined for this costing method, are those costs that have to be borne by the laboratory whether a few or many tests are performed, and the total cost of processing specimens may be represented by the sum of the factors listed in table 1 . These costs are apportioned across the annual workload for any particular test. Workstations were identified as an area of the laboratory where a defined workforce, which may be comprised of whole-time or part-time staff, or both, produce defined products, either as laboratory tests, or processes contributing towards tests. In recognition that all resources have a cost contribu-

Table 1 Fixed cost of processing specimens

Medical laboratory scientific officer time Clerical and secretarial time

Medical staff time

Scientific staff time

Capital equipment and capital charges Overheads tion the workforce salary at a workstation must be apportioned across the processes or products that derive from that workstation. The Datatree costing method helps in apportioning that cost (figure). WELCAN ${ }^{5}$ units are accumulated at each workstation and divided into the workstation salary cost to give a cost/ WELCAN unit. By multiplying this unit cost by the WELCAN weighting of the product and the annual numbers of product produced, a contribution to the fixed costs is obtained. WELCAN units are not designed for costing purposes but in this instance are used as a weighting system to allocate costs across tests proportionately with no reference to their use as measurements of work time. Others have suggested that they may be used in this way. $^{6} 7$

Datatree is configured with cost and tree sections, together with tree representations of the contributing costs, providing an easily understandable way of demonstrating the costing method to colleagues. All items paid for by the laboratory are entered into a cost section and then apportioned between trees. Once the files are complete it is easy to generate reports for purchasers from the report generator using one of the predefined formats or by development of individualised formats.

Overheads are accumulated in a tree and apportioned across tests. Two separate overhead trees might by considered: one for apportioning by test weighting and the other by test requests without any weighting. By this method, overheads such as the cost of a laboratory computer system could be spread across tests in proportion to their computer usage, whilst other overheads such as the salaries of clerical staff could be evenly apportioned across all tests. Examples of overheads to be considered are shown in table 2 .

Consumables are placed in "cost sections" and a cost can be immediately attributed to the consumables from the items invoice. A record factor associated with the consumable can divide the cost of the consumable so it can be utilised in a "tree section," so that if blood 
Table 2 What are overheads?

\begin{tabular}{l} 
Hospital overheads \\
Energy \\
Water \\
Heating \\
Building size \\
Maintenance \\
Contribution towards: \\
Administration \\
$\quad$ Personnel \\
$\quad$ Estates office \\
$\quad$ Engineering \\
Cleaning services \\
$\quad$ Laundry \\
Laboratory overheads \\
Training \\
Research \\
Accreditation \\
Quality control \\
Laboratory computer \\
Administrative personnel \\
Telephone costs \\
Phlebotomy \\
Contribution towards: \\
Transport system \\
Portering system \\
\hline
\end{tabular}

count bottles are bought in boxes of 2240 then the record factor would be $1 / 2240$ to allow a single blood count bottle to be used in the building of a tree. The cost of 2240 blood count bottles would likewise be divided by 2240 and this cost attributed to one bottle. Wastage and quality control samples can be accounted for by the system by applying a mark-up on the consumables used. No markup would be required for the fixed costs as the wastage and quality control samples do not contribute to the revenue of the laboratory.

Haematology and blood transfusion Of the four disciplines this is the most straightforward. Workstations are easily defined and the number of products and processes are not large. The above costing method is readily applicable.

Chemical pathology Weighting units are defined for the pre-analytical, analytical, and post-analytical phases of processes/products. The pathologist can attribute his or her own weighting units to the tests provided based on a composite of the WELCAN units. An alternative method is for the pre-analytical preparation to be attributed to an overhead tree and apportioned across all tests.

Microbiology The main problem is that a variable amount of work is done on specimens of the same type. To allow for this and to be able to provide a single cost a composite WELCAN value was used. This was calculated by recording the work done on 100 specimens of each type, summing the associated WELCAN values and dividing the total by 100 . This will obviously produce weightings specific to each laboratory.

The media room output is fed into several, but not all, workstations. The proportion of media used at each workstation needs to be estimated.

Histopathology Histopathology departments perform one investigation (a histopathological examination) on possibly in excess of 300 types of tissue specimen. Coupled with the fact that the work done on each specimen may vary considerably in any department because of local working practice, the problems are compounded. In histopathology fixed and variable costs do not feed directly into the specimen trees, but rather into subtrees created to cost procedures, such as staining, tissue processing, etc. These processes can be weighted depending on previous workload and local practice, producing costs which reflect the complexity of a histological investigation in a given department.

Cervical cytology can be treated similarly, the procedures feeding into trees which take into account two-smear cases, hormonal evaluation, abnormal recall, as well as positive and negative smears, thereby producing a single volume sensitive cost for a cervical smear.

Specialised procedures such as electron microscopy, immunocytochemistry, and resin processing techniques, are all catered for by this costing method.

\section{Summary}

The Datatree costing project in Wales has provided the Welsh pathology laboratories with a standard costing package that allows pathologists to understand how their own laboratory's test costs are compiled. The software provides answers to the question "what if?..." and shows instantly the effect of salary or consumable cost alterations. Resource management at a laboratory level is enhanced by a greater knowledge of costs, particularly in relation to volumes of work. Perhaps this is one of the stepping-stones across the river to the "open market." In the United Kingdom NHS any information of this kind must be regarded as invaluable.

1 Stilwell JA, Woodford FP. Microcomputer software to facilitate costing in pathology laboratories. $f$ Clin Pathol 1987;40:817-25

2 Audit Commission. The Pathology services. A management review. London: HMSO, 1991.

3 Dick HM. Costing of Pathology services in the United Kingdom National Health Service. $f$ Clin Pathol 1991;44:705-9.

4 Bain J. Budget holding: the first 150 days in Calverton. BMF 1991;303:907-8.

5 WELCAN Secretariat. WELCAN UK workload measurement system manual. London: HMSO, 1990.

6 Tarbit IF. Laboratory costing system based on number and type of test: its association with the WELCAN workload type of test: its association with the WELCAN wo.
measurement system. $\mathcal{F}$ Clin Pathol 1990;43:92-7.

7 Otter PI, Pease AA. A computerised spreadsheet for audit of a microbiology laboratory. Med Lab Sci 1991;48: 217-24. 